\title{
Didáctica artística y medioambiental: la estampación de materias vegetales como recurso educativo
}

\section{Artistic and environmental didactics: stamping of vegetal matter as a teaching resource}

\author{
José Víctor Villalba Gómez ${ }^{1}$ \\ josevictor.villalba@um.es \\ Universidad de Murcia, España
}

\section{Resumen:}

En esta investigación se muestra un proyecto interdisciplinar que enlaza competencias, valores y contenidos a partir de la unión de enseñanzas artísticas y medioambientales. El objetivo principal es crear un método didáctico basado en la interacción del arte con espacios naturales destacados, teniendo el grabado, el color y las materias naturales como principales vehículos técnicos del proceso de enseñanza aprendizaje. Estas materias, tales como hojas, tallos e incluso frutos, no suelen ser aprovechadas por los agricultores o por los responsables de mantener en buenas condiciones las zonas medioambientales protegidas. El contexto se sitúa en la formación universitaria y plantea un discurso de innovación unificando los conceptos de educación artística y educación ambiental a través de una educación transversal para trabajar diferentes áreas del currículo. La línea de trabajo está denominada "planificaciones artísticas ecológicas". Todo ello enfocado para alumnos del Grado de Educación Primaria, futuros

\begin{abstract}
:
In this study we aim to show an interdisciplinary project that links competences, educational values and content from artistic and environmental teaching. The main objective is to create a teaching method based on the interaction of art with outstanding natural areas; taking engravings and colours and natural materials as the main vehicles of the teaching-learning process. These materials, including leaves, stems and even fruits, are not usually used by farmers and workers in charge of keeping protected natural areas. The context of the study is university education and we propose an innovative project that transversally couples the concepts of artistic and environmental education with a view to working with different areas of the curriculum. The line of work is called "ecological artistic planning." The project is aimed at primary education students, future teachers, that through field trips and classroom experimentation with graphicart techniques such as monotype printmaking construct and relate elements of
\end{abstract}

\section{Dirección para correspondencia (correspondence address):}

José Víctor Villalba Gómez. Dpto. Expresión Plástica Musical y Dinámica. Facultad de Educación. Universidad de Murcia. Campus de Espinardo, s/n. 30100 Espinardo (España). 
Didáctica artística y medioambiental: la estampación de materias vegetales como recurso educativo

José Víctor Villalba Gómez

maestros, que a través de salidas de campo y experimentación en el aula con técnicas grafico-plásticas de grabado como el monotipo, llevan a cabo un método de construcción y relación de elementos del lenguaje visual y plástico con materiales extraídos del propio entorno natural, sin alteraciones del mismo. En dicho trabajo, se analizan los contenidos y el proceso como estrategias instrumentales de la competencia artística que han de adquirir los alumnos para su futura labor docente. Los resultados del experimento se reflejan en forma de vivencias y creación de composiciones plásticas muy diversas, construidas individualmente, pero realizadas al unísono por todos los participantes en un clima de diálogo entre los integrantes y el propio entorno. Finalmente, en pleno contacto con la naturaleza, los hallazgos plásticos y curriculares son tratados en una asamblea con los trabajos expuestos.

\section{Palabras clave:}

Competencia artística; educación ambiental; materia vegetal; monotipos; futuros formadores. visual and plastic language with materials extracted from the natural environment without altering it. In this article we analyse content and processes as instrumental strategies of the artistic skills that students should acquire for their future teaching. The results of the experiment are reflected in experiences and different plastic compositions which, despite being built individually, all the students did at the same time in an atmosphere of dialogue among them and the environment. Finally, in full contact with nature, the plastic compositions are displayed and curricular findings are discussed in an assembly.

\section{Key words:}

Artistic competence; environmental education; plant matter; monotypes; teacher training.

\section{Résumé:}

Cette recherche montre un projet interdisciplinaire qui combine des compétences éducatives, des valeurs et des contenus à partir de l'union des enseignements artistiques et environnementaux. L'objectif principal est de créer une méthode d'enseignement basée sur l'interaction de l'art avec des espaces naturels remarquables; prenant la gravure, la couleur et les matériaux naturels comme les principaux outils du processus enseignement-apprentissage. Ces matériaux, les feuilles, les tiges ainsi que les fruits, ne sont habituellement pas exploités par les agriculteurs et par les responsables de la maintenance du bon état des lieux environnementaux protégés. Le contexte est situé dans l'enseignement universitaire et propose un discours d'innovation qui associe les concepts de l'éducation artistique et de l'éducation environnementale à travers l'éducation transversale dans les différents domaines du programme éducatif. La ligne de travail est appelée " planification artistique écologique ».. Focalisée entièrement autour des études pour être enseignant à l'école primaire, les futurs enseignants effectuent une méthode de construction et de rapport aux éléments du langage visuel et plastique avec des matières extraites du milieu nature sans les altérer, qui à travers des sorties pédagogiques du terrain et de l'expérimentation dans la classe, en employant des techniques graphiques-plastiques telles que l'impression monotype. Dans ce travail sont analysés le contenu et le processus comme stratégies instrumentales de la compétence artistique que les étudiants doivent posséder comme futurs enseignants.

Les résultats de l'expérimentation sont montrés sous forme d'expériences et de création de compositions plastiques très diverses, construites individuellement, mais interprétées à l'unisson par tous les participants dans un climat de dialogue entre les membres et le propre environnement.

Finalement, les découvertes plastiques et des programmes d'études sont traités dans une assemblée en plein air et en présence des œuvres exposées. 


\section{Mots clés:}

Compétence artistique; éducation environnementale; matière végétale; monotypes; futurs professeurs.

Fecha de recepción: 23-2-2016

Fecha de aceptación: 28-6-2016

\section{Introducción}

La competencia artística es una de las competencias básicas menos consideradas dentro del panorama educativo institucional, un proceso de minorización curricular que ha venido intensificándose con las últimas leyes educativas (LOMCE). Es por ello que, en la docencia universitaria, la responsabilidad docente del personal encargado de impartir materias artísticas de los futuros maestros, no se limita a enseñar métodos para impartir conocimientos y destrezas artísticas, sino que se crea la necesidad de enfocar la materia de forma instrumental y transversal haciendo uso de un pensamiento divergente, hilando a través de proyectos artísticos diferentes contenidos y conceptos, extrapolando métodos de creación y expresión artística al terreno didáctico, trabajando por un aprendizaje significativo.

La observación y el análisis que se puede realizar del entorno, es un pilar fundamental para programar y crear situaciones y procesos de enseñanza-aprendizaje. Por tanto, pensamos que es posible crear planificaciones en las que es el propio paisaje y los elementos que le abastece, el que ofrece posibilidades didácticas ideales para trabajar los contenidos sin obviar las necesidades y valores imprescindibles en la sociedad actual, especialmente el respeto y cuidado de la naturaleza.

Además, en esta propuesta se ponen a prueba las capacidades creativas e interpretativas del alumnado, se fomenta la curiosidad por conocer nuestro entorno, especialmente el natural y sus aspectos curriculares fundamentales fuera de las aulas y nuestros hogares. No debemos de obviar que muchas de las personas no lo conocen más allá de la información alcanzada a través de las grandes superficies y los diversos canales que ofrecen los medios de comunicación. 
Didáctica artística y medioambiental: la estampación de materias vegetales como recurso educativo

José Víctor Villalba Gómez

\section{Marco teórico}

\section{Educación ambiental y centros de interpretación}

En nuestra línea de trabajo existe una clara conexión con otros centros e instituciones como las Asociación Española de Educación Ambiental (AEEA, 2014), el Centro Nacional de Educación Ambiental (CENEAM, 2014) y diversos centros educativos repartidos por toda la geografía nacional como los ecocentros y ecoescuelas, en los que proponen, promueven y llevan a cabo iniciativas y acciones curriculares que trabajan con las emociones e integran la educación ambiental con contenidos de distintas áreas de conocimiento, produciendo así una renovación pedagógica (Villalba y Flores, 2015).

Nuestra investigación tiene relación todavía más directa con proyectos e iniciativas que realizan espacios museísticos como los centros de interpretación, especialmente los dedicados a la Naturaleza. Algunos de los ejemplos más significativos son: centro de interpretación Racó de I'Olla del parque de la Albufera de Valencia, centro de interpretación del parque de las tablas de Daimiel, el parque de las ciencias de Granada, el centro de interpretación en el valle del Jerte, el centro de interpretación de la floración de Cieza y otros centros de interpretación dedicados al reciclaje, así como yacimientos arqueológicos, etc. Todos ellos realizan actividades utilizando competencias artísticas en atención a temáticas relacionadas con el agua, la biodiversidad, la flora, la fauna, el reciclaje, la arqueología, etc.

La función de este tipo de centros es la de impulsar un ambiente creativo, incentivando al público de manera atractiva la curiosidad por conocer aspectos vitales de la sociedad, su patrimonio medioambiental y cultural, y su responsabilidad por asegurar la continuidad. Las labores que se desarrollan son de investigación, conservación y divulgación. Estas instituciones, públicas o privadas, adquieren el nombre de interpretación porque traducen el lenguaje técnico y complejo del legado que representan de forma comprensible y sencilla a todos los públicos.

Interpretar puede entenderse entonces como el arte de presentar al público un lugar o un objeto, o un conjunto de ellos, para informarlo, entretenerlo y motivarlo al conocimiento. El fin de la interpretación es dejar en el visitante un entendimiento de por qué y en qué sentido es importante ese lugar y los objetos que se exponen (Santacana, 2005, p. 63). 
El objeto principal de los centros de interpretación es transmitir sensibilidad, conciencia, entendimiento, entusiasmo, compromiso, etc. Para llegar a tal fin, los centros no solo muestran el material objeto de interpretación, sino que revelan al público el significado e interrelación del patrimonio. Para tal fin utilizan recursos expositivos y didácticos que hacen participar al espectador en su propio aprendizaje, para que inicie un viaje acompañado en el que junto a los medios didácticos propuestos aportados por el centro, objetos y personal orientador; sea el propio visitante el encargado de descubrir el conocimiento (Santacana y Serrat, 2005).

Los centros de interpretación suelen situarse en lugares que tienen relación directa con los contenidos que se tratan: edificios históricos o parajes donde se edifican y se crean instalaciones normalmente respetuosas y acordes con el lugar, con su entorno.

La tipología de los centros es muy variada: arquitectura, yacimientos arqueológicos, centros etnográficos, bodegas, salinas, minerías, prehistoria, trashumancia y agua, fauna y flora específica, espacios naturales protegidos, patrimonio rural, etc. Precisamente son estos últimos los que por sus contenidos tienen absoluta relación con nuestra propuesta, por lo que partimos de ellos para crear propuestas artístico-didácticas.

La existencia de todos los tipos de centros nombrados hasta ahora, demuestra que existe un compromiso ético y que, con gestos y acciones, se están intentando divulgar el patrimonio material e inmaterial. Sin embargo, los centros didácticos que más nos interesan son los que intentan abolir problemas medioambientales. Actualmente existen unos 300 centros repartidos por toda la geografía nacional, quizás no son demasiados, pero esta cifra indica que se está gestando una considerable cultura de educación ambiental y sostenibilidad. Una cultura que nos afecta a nosotros como educadores e investigadores, ya que no somos ajenos a los grandes problemas culturales y medioambientales. Por tanto, consideramos la necesidad de crear más lazos de unión entre este tipo de centros y los centros de formación reglada (colegios, institutos y universidad), viendo en estos recursos un pilar importantísimo en la formación, transgresión y adquisición de compromisos y valores de respeto y convivencia con el medio natural para la formación integral del niño: el futuro empresario, al futuro arquitecto, al futuro político, al futuro trabajador, etc. Si al mismo tiempo se hace desde una perspectiva artística, podremos ser capaces de universalizar todos aquellos valores en los que 
Didáctica artística y medioambiental: la estampación de materias vegetales como recurso educativo

José Víctor Villalba Gómez

los educadores insistimos tantas veces a través de la creación, valorando el medio, valorando el patrimonio, así como valorando a los demás.

Las acciones y premisas fundamentales que promueven los centros de interpretación, son integradas en nuestra propuesta. Como veremos más adelante, se propone un proyecto que parte de términos como el grabado, el reciclaje y la ecología, planteando recursos didácticos muy concretos, pero en el que existen distintas variables prácticas.

Una característica clave del arte actual y que utilizamos en la investigación es el apropiacionismo. Estos términos tienen su origen en artistas como Pablo Picasso o Marcel Duchamp. En el primero destacan los conceptos de collage y ensamblaje. Sin embargo, el segundo descubre los conceptos de "readymade" u "objet trouvé" (Arte Encontrado). Ambos artistas realizaron obras haciendo uso de objetos que no tienen una función específicamente artística y que se recontextualizan para conseguir tal fin (Moreno, Abad y López, 2011), un fin artístico y didáctico en nuestro caso.

\section{El arte y su capacidad interactiva}

El carácter lúdico e interactivo del arte, intrínseco en la transmisión de conocimientos, valores y competencias; permite ilimitadas posibilidades expresivas y didácticas debido a la versatilidad de los materiales y entornos de creación, hábiles para trabajar cualquier concepto. No menos posibilidades expresivas y didácticas ofrecen las materias propias del entorno natural.

En el arte posmoderno, a finales de los años sesenta, surgieron ismos como el Land Art o Earthworks, en los que para artistas como Richard Long o Robert Smithson, el propio paisaje y sus elementos transformados ofrecían herramientas y conceptos vinculados a nuestro profundo arraigo con la naturaleza (Tatarella, 2014). También obras como las de Giuseppe Pepone, ofreciendo diálogos en plena naturaleza mediante la manipulación vegetal. Diálogos que, en la mayoría de los casos, entran en un estado muy poético y reflexivo. Tanto es este diálogo que a nivel educativo pueden ofrecer grandes posibilidades interactivas (AA.VV., 2014). Estos movimientos artísticos surgieron como respuesta a la industrialización y urbanización en Inglaterra y EE.UU. Posteriormente y hasta nuestros días, existen otras manifestaciones con una profunda carga conceptual ligada a la naturaleza y a los graves problemas medioambientales, todas ellas englobadas en el arte ecológico donde los materiales arcaicos como las 
nuevas tecnologías, se enlazan para reflexionar y mostrar a la sociedad la realidad medioambiental y posibles soluciones.

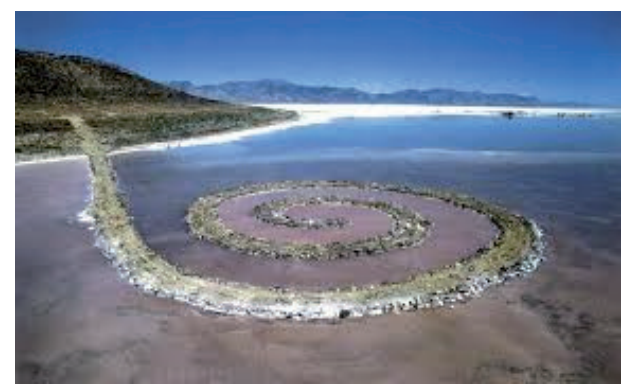

Figura 1. Spiral Jetty de Robert Smithson.

Las característica principales del arte posmoderno son dos: el apropiacionismo y la descontextualización, atribuidas al artista dadaísta Marcel Duchamp y causada indirectamente por los avances socioeconómicos y tecnológicos de los años cincuenta. Sin embargo, estas características no son cambios exclusivos del mundo artístico, sino que también se extrapolan a la pedagogía (Moreno y Abad, 2011). Todo ello sin olvidar claros antecedentes que hilan arte y pedagogía como Friedrich Fröbel, que a mediados del siglo XIX, ya puso de manifiesto la función pedagógica de los objetos y materias naturales en el propio entorno. Lo mostró con el concepto que acuñó kindergarten en el que desarrolló material didáctico para niños, considerados como los principales protagonistas de su propia educación a través del descubrimiento e interacción con los materiales naturales (Böhm, 2010).

Más allá de los inicios pedagógicos de la intervención con la materia y el paisaje en la expresión plástica, queremos resaltar una serie de proyectos enfocados a la Educación Primaria y Secundaria.

Un proyecto pionero de concienciación ecológica en el ámbito de la educación plástica fue "Art and the Built Environment (ABE), arte y entorno construido, llevado a cabo en Gran Bretaña a finales de la década de los 70 y principios de los 80. Los objetivos, contenidos y métodos de este proyecto causaron una gran influencia en la educación artística mundial. ABE partió de esta problemática urbana y quiso trabajar cuestiones asociadas al urbanismo y al interés social por una ciudad sana. Aunque estos aspectos no llegaron a ser desarrollados con toda la potencialidad deseada, crearon una conciencia traducida con los años 
Didáctica artística y medioambiental: la estampación de materias vegetales como recurso educativo

José Víctor Villalba Gómez

en retos de urbanismo. Estos retos son la recuperación de espacios públicos y de la vida callejera, las posibilidades estéticas y sensoriales del diseño urbano o la participación ciudadana. Tenemos constancia de este trabajo gracias a Educación Artística y Ambiental (EAA) del profesor e investigador Alfredo Palacios (2006), un proyecto posterior que estudia el caso británico. EAA es un trabajo que reflexiona acerca de las aportaciones que la educación artística puede ofrecer a la educación ambiental, considerando como punto de partida la relación ser humano- medio ambiente y, en particular, la dualidad entorno natural-ciudad. Es decir, la idea de generar un acercamiento interdisciplinar que une arte, arquitectura, ecología, sociología, activismo social, etc. En definitiva, disciplinas que estando relacionadas con el mundo educativo, podrían transformar nuestro entorno en otro más acorde con la realidad global.

Un trabajo en la línea de Palacios (2006), es el que lleva acabo Arno Rafael Minkkinen (2015), profesor y artista finlandés que a través de fotografía como medio expresivo, propone proyectos artístico-educativos en los que es el propio cuerpo humano el que se fusiona con el entorno, actuando como vínculo conciliador, formando parte del paisaje de manera totalmente integrada. A diferencia del proyecto de Palacios (2006), que trabaja en el paisaje urbano (2006), Minkkinen utiliza el paisaje en su estado más natural, trabajando en bosques, lagos y otros escenarios donde es posible crear diálogos entre nuestro cuerpo al encontrarse en continua interacción con el medio y los materiales que este nos ofrece.

\section{Técnicas de grabado e impresión}

El grabado es una disciplina artística en la que el creador utiliza diferentes técnicas de impresión, que tienen en común el hecho de plasmar una imagen sobre una superficie rígida, Ilamada matriz, dejando una huella que después alojará tinta y será transferida por presión a otra superficie como papel o tela, lo que permite obtener varias reproducciones de la estampa. El proceso de transferir la tinta al papel se denomina estampación o impresión.

Existen diferentes tipos de estampación, normalmente ligados a los materiales y a los procesos de grabación. Los métodos tradicionales de impresión hacen uso de la presión mediante técnicas manuales como el frotado, mecánicas con tórculos y prensas o con distintos medios como paneles o cucharillas (Bernal, 2013). 
Con las técnicas de grabado es fácil controlar repetidamente similares o idénticas impresiones gráficas: puntos, líneas, formas, texturas y color (Garrido, 2014).

La etapa con mayor vigor de las técnicas de grabado y estampación se corresponde al periodo del Renacimiento en Italia (siglos XV y XVI). Sin embargo, desde hace tres mil años en Mesopotamia ya utilizaban técnicas de reproducción utilizando la piedra como matriz, tallando en piedras circulares que posteriormente hacían rodar sobre materia arcillosa en la que quedaban grabados los diseños. Entre esta sedentaria civilización y la época renacentista, en China y en Japón (siglo II d.C. en adelante), tras la invención del papel se realizaron impresiones utilizando la piedra como molde. Los primeros grabados europeos se realizaron sobre soporte textil en el siglo VI (ibídem).

Disponemos de numerosas técnicas de grabado y métodos gráficos indirectos: grabados en relieve como la xilografía y linografía (madera y linóleo respectivamente), grabados en hueco como el agua fuerte, la aguatinta (por efecto de la mordida al motriz metálica del ácido), al buril, punta seca o mezzotinta (métodos directos), y otras técnicas como el barniz blando, la tinta china con azúcar, la litografía (piedra), esmaltografía, la monotipia y técnicas más actuales como el fotograbado y el electrograbado (ibídem).

Por otra parte, existen otras técnicas muy actuales como el grabado matérico, donde el leit motiv de la experimentación plástica reside en la manipulación de las pastas papeleras para la construcción de obras.

Sin embargo, son de especial interés en este trabajo aquellas técnicas de impresión utilizadas como recurso científico en la documentación científica, como por ejemplo las que hacen uso del frotado manual, con cucharillas, como sucede con las eptipas, originarias de Japón. Destacamos estas últimas por guardar extrema similitud con las producciones artísticas de nuestras actividades. Según el documento "dibujo científico: arte y naturaleza, ilustración científica, infografía, esquemática", en el que se muestra la evolución de las artes plásticas como medio de documentación científica; en la documentación de especies botánicas, haciendo referencia a las eptipas, ya eran utilizadas como recurso documental antes del descubrimiento de la estampación a color por procedimientos mecánicos (Cabezas, López, Oliver, Campos y Barbero, 2016, p. 249).

Por otra parte, existen técnicas de estampación en las que son obje- 
Didáctica artística y medioambiental: la estampación de materias vegetales como recurso educativo

José Víctor Villalba Gómez

tos cotidianos los que actúan como propias matrices. Esta técnica tiene su origen en los fósiles, en los que la naturaleza se ha encargado a lo largo de millones de años de transformar materias orgánicas en positivos y negativos a través de la materia mineral. Lo mismo ocurre con la técnica del Gyotaku (gyi-tah-koo, frotar y pescado) una técnica de estampación muy extendida en Japón trabajada con fines didácticos en escuelas, que se originó a mediados del siglo XVIII cuando a un pescador se le ocurrió estampar en papel los propios peces para tener un recuerdo físico de sus mejores capturas (Pardo, 2011). También ocurre un fenómeno plástico similar cuando caen las hojas y otros elementos orgánicos al suelo. Con el tiempo dejan una huella sobre la tierra fina en la que de manera efímera se crea un entramado de formas y texturas, un grabado natural.

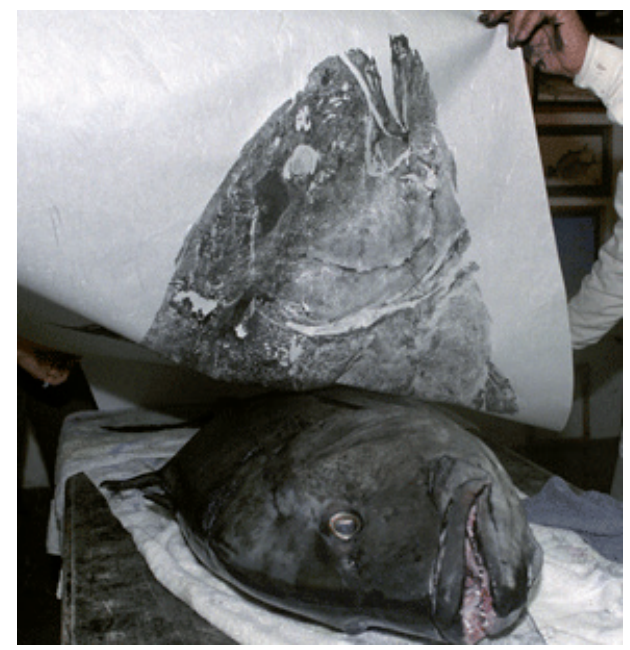

Figura 2. Estampación con la técnica del gyotaku, 2011.

En muchas ocasiones es la propia naturaleza la que nos ofrece aspectos plásticos y didácticos. Simplemente hay que observar dichos aspectos, analizarlos y planificar su didáctica para enseñar con ellos como actualmente se hace con el Gyotaku en Asia. 


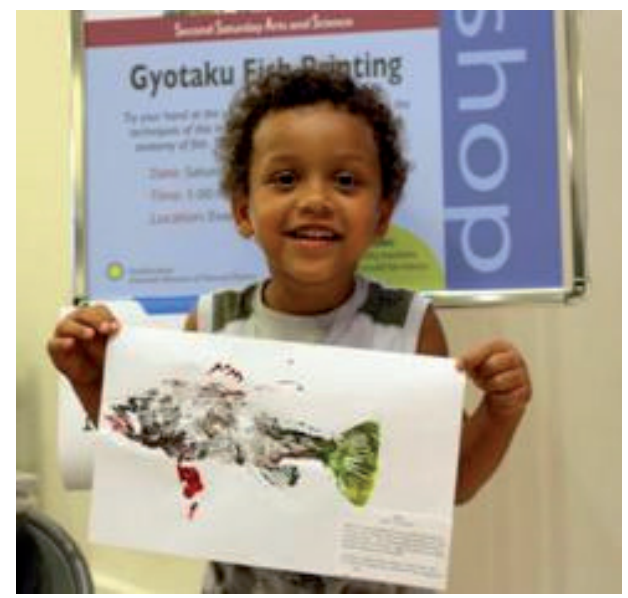

Figura 3. Adaptación del gyotaku a niños, 2011.

\section{Marco empírico}

\section{La estampación como herramienta didáctica de interacción y construcción del paisaje}

En la línea de investigación que se plantea, hemos realizado un proyecto en el que proponemos a estudiantes del Grado de Educación Primaria un proceso de enseñanza-aprendizaje fusionando actividades en el aula con otras realizadas en salidas programadas en el entorno natural, uniendo la educación formal con la informal. El objetivo principal es mostrar multitud de aspectos didácticos que se trabajan al utilizar los medios que ofrece la naturaleza si aplicamos recursos artísticos (materiales y procesos). Las competencias de nuestra propuesta que trabaja el alumnado son:

Competencia básica: aprender a fomentar la observación y sensibilización con el medio natural.

Competencia general: conocer y utilizar distintos métodos didácticos a través de la naturaleza y el arte.

Competencia específica 1: reflexionar acerca de las posibilidades didácticas de los materiales naturales y su entorno.

Competencia específica 2: enseñar a expresar plásticamente con elementos grafico-plásticos.

Competencia específica 3: conocer y trabajar con herramientas, re- 
Didáctica artística y medioambiental: la estampación de materias vegetales como recurso educativo

José Víctor Villalba Gómez

cursos y procesos plásticos para su utilización con fines didácticos en un medio natural, aplicados en la Educación Primaria e Infantil.

A lo largo de la propuesta se impartieron las siguientes materias:

1- Arte y Naturaleza. Posibilidades Didácticas

2- Didácticas artísticas en el medio natural y urbano. Diferentes proyectos.

3- El grabado y el color como recurso educativo.

4- Interacción artística en el medio natural. Utilización de recursos naturales en el grabado: creación de obra artística.

5- La exposición como recurso didáctico.

Las materias 1, 2 y 3 tienen un carácter contextualizador para dar soporte teórico y de preparación técnica con el fin de abordar las materias 4 y 5 . En estas últimas, es el alumnado el principal protagonista de su aprendizaje. Una vez analizados los materiales recogidos en el paraje natural, el alumno manipula los materiales proporcionados en continuo razonamiento y feedback con los compañeros y personal docente a través de debates y el mismo proceso creativo, donde se cruzan conocimientos ambientales y pictóricos a través de la estampación de las plantas.

En el proyecto se ha planteado a los alumnos la adaptación y utilización de técnicas artísticas en un entorno con materias naturales muy concretas: tallos y hojas de plantas del entorno del río Segura y su huerta.

Concretamente, las técnicas artísticas que utilizamos pertenecen a disciplinas del grabado y pintura: la estampación (medio indirecto) y la pintura acrílica (medio directo). Ambas técnicas son unificadas en un soporte único de papel; grandes pliegos de papel blanco y grueso (se ha utilizado papel Canson liso de $300 \mathrm{~g} / \mathrm{m}$ y de $700 \times 1000 \mathrm{~mm}$ ), aunque podrían utilizarse otros papeles que aporten granulado, color y otros grosores más finos.

Las herramientas utilizadas han sido: brochas, pinceles de pelo vegetal, pulverizadores de agua, bayetas absorbentes, tablas de DM plastificadas y sin plastificar de $500 \times 500 \times 8 \mathrm{~mm}$.

Antes de describir el proceso, es necesario tener conocimientos básicos del uso del color, composición y tipos de elementos del lenguaje visual y plástico. Estos conocimientos se aportan en el aula en las materias 1,2 y 3 . 


\subsection{Fases del proceso}

El primer paso que se realizó fue analizar en el aula las posibilidades didácticas que ofrecía el proyecto, revisando el currículum actual de Educación Primaria y estableciendo relaciones del medio natural y sus elementos vegetales con diferentes áreas de conocimiento.

En segundo lugar, se realizó un desplazamiento del alumnado a un paraje emblemático del medio natural, concretamente a la zona de ribera del río Segura y acequias con norias en la localidad murciana de Abarán, una de las ciudades que todavía conserva sus huertas con regadíos tradicionales, donde se realizó una toma de contacto con el paraje y las especies vegetales, donde además se visitó una huerta hortofrutícola con la presencia de su propietario.
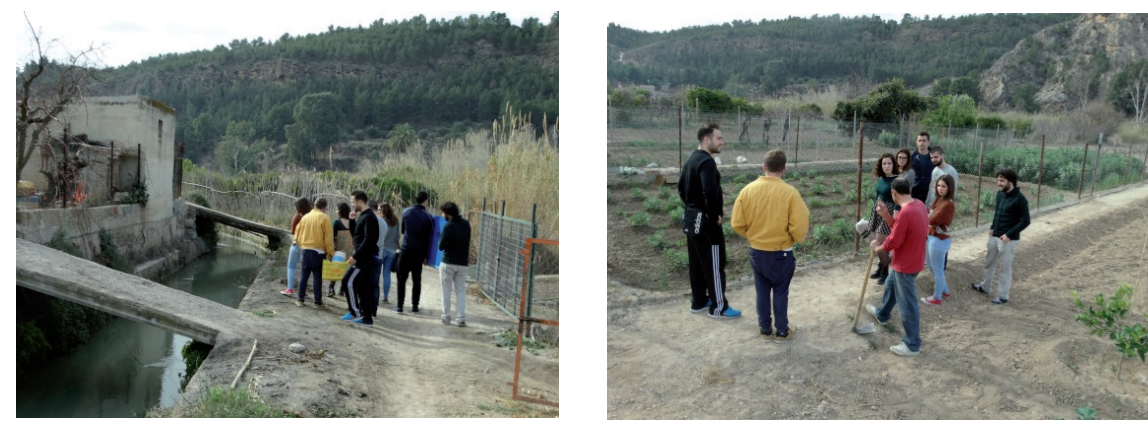

Figura 4 y 5. Inspección del entorno junto a una de las acequias de Abarán.

En tercer lugar, se recogieron cuidadosamente tallos y hojas de algunas de las especies de la ribera y de la huerta visitada. Con respecto a esta última, previamente se realizó una clasificación de las distintas especies vegetales: cítricos (limonero, naranjo, pomelero y mandarino), frutas de hueso (melocotonero, paraguayo, albaricoquero, ciruelo, nectarino, almendro, palmera datilera, níspero, etc.), frutales de pepita (manzano, peral, membrillero), verduras (apio, alcachofa, acelga, espinaca, coliflor, brócoli, col, tomatera, berenjena, pimiento, melonera, pepino, etc.) y tubérculos (zanahoria, cebolla, ajo, patata, etc). En esta fase, destaca un dato significativo, la alta curiosidad mostrada por los alumnos por conocer el origen de muchos frutos, pues resulta paradójico que un buen número de los alumnos no conociera algunos de los frutos o simplemente manifestara tener constancia de su existencia al verlos en la sección de frutas y horta- 
Didáctica artística y medioambiental: la estampación de materias vegetales como recurso educativo

José Víctor Villalba Gómez

lizas de los supermercados. Es por ello que in situ, solicitamos la intervención de un agricultor que explicó al grupo acerca del tipo de regadío de la zona, los tipos de plantas cultivadas, la climatología óptima para los tipos de plantas y las labores que ejercen a lo largo de los periodos estacionales.

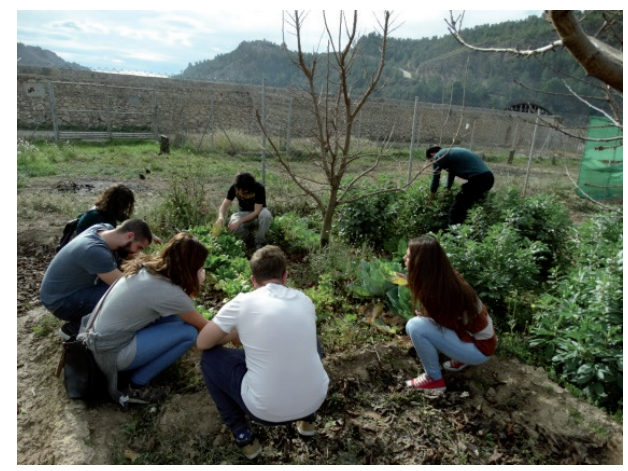

Figura 6. Inspección de las planas y recogida de tallos y hojas.

Posteriormente, el alumnado analizó las formas y texturas de los vegetales, seleccionando un elemento principal y dos secundarios para estampar. A continuación, se reflexionó acerca de aspectos compositivos tales como: formato, color, texturas, formas, volumen, dirección, contraste, saturación, peso de los elementos. También se analizaron los aspectos didácticos relacionados con las acciones efectuadas hasta ese momento y las que se llevarían a cabo más tarde.

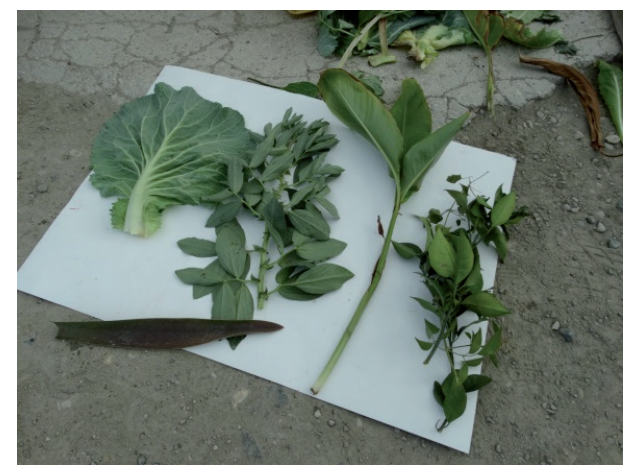

Figura 7. Selección de tallos y hojas.

Seguidamente, se realizaron estampaciones con las partes de las especies vegetales recolectadas siguiendo el procedimiento que a continuación exponemos: 
Se procede al manchado e impregnación de hojas o tallos con pintura acrílica diluida con diferentes proporciones de agua. Para ello, se coloca la pieza vegetal sobre una tabla o soporte impermeable (tabla de DM plastificada). Por otro lado, se precisa de otro soporte tipo paleta (tabla de DM) donde las brochas y pinceles se impregnan de color, que una vez diluido y habiendo realizado las mezclas oportunas, se aplicará sobre las plantas.

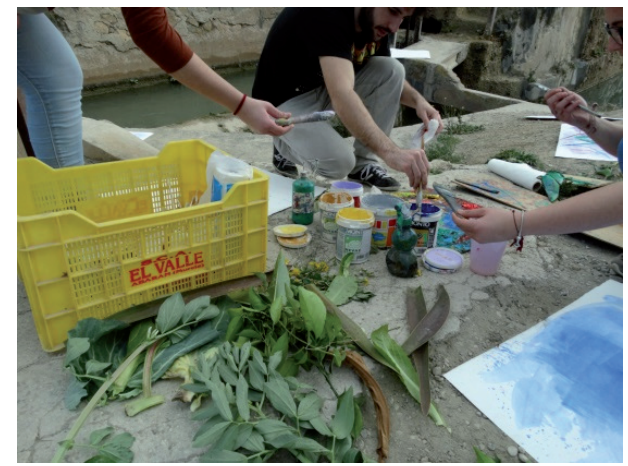

Figura 8. Proceso de realización de monotipos.

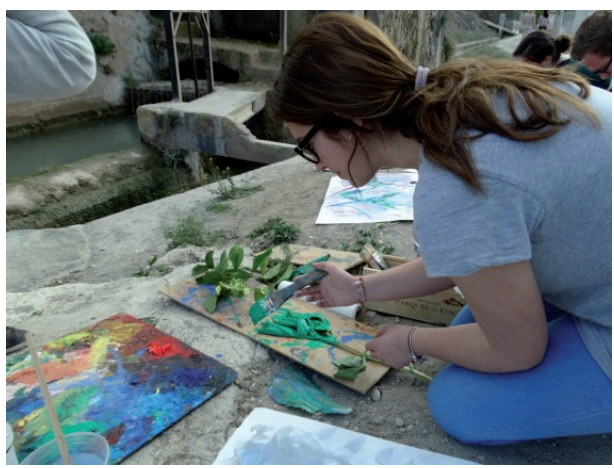

Figura 9. Proceso de realización de monotipos.

El soporte puede ser papel o tela y debe acondicionarse previamente para la estampación. Para su preparación, se humedece con agua el soporte utilizando un pulverizador y/o brochas. A continuación, con pinceles y brochas el color, bastante diluido, se proyecta sobre el papel mojado, configurando degradados de manera bastante acuarelada, aportando atmósferas de color.

El paso sucesivo es realizar la estampación del vegetal sobre la su- 
Didáctica artística y medioambiental: la estampación de materias vegetales como recurso educativo

perficie de trabajo. Existen diferentes modos de realizar el estampado: estampación tradicional (a mano o con presión añadida mecánicamente por un tórculo o prensa) y estampación a base de impactos y frotados. Para este fin se coloca la parte de la planta impregnada de acrílico sobre el papel húmedo y colorido. Posteriormente, se coloca la tabla sobre el papel y con las propias manos se presiona, separando la tabla y los elementos vegetales del papel pocos segundos después. En este caso se han utilizado las plantas como herramienta para plasmar las formas y texturas sobre el soporte.

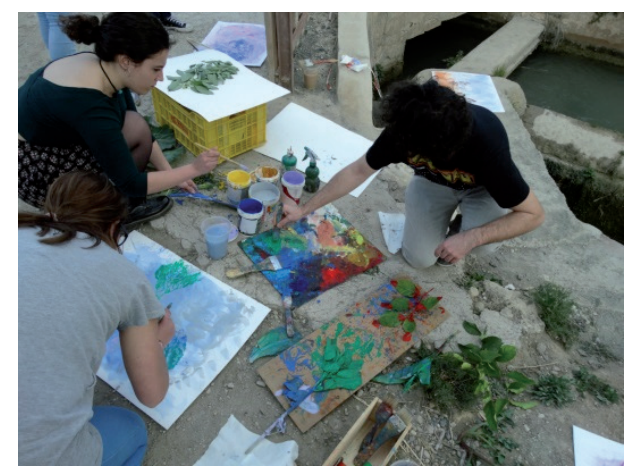

Figura 10. Proceso de realización de monotipos.

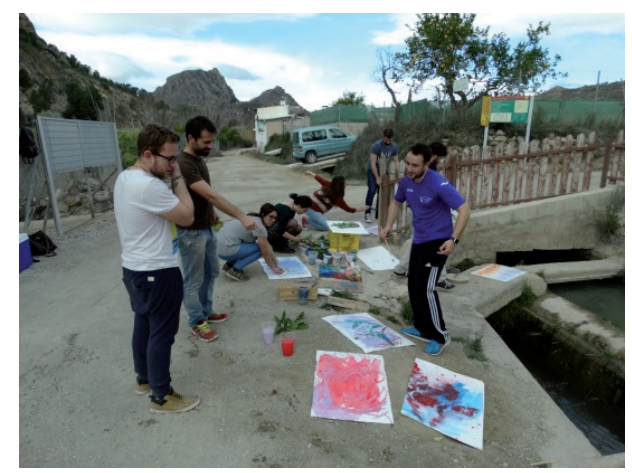

Figura 11. Proceso de realización de monotipos.

Existen grandes variantes y posibilidades en la elección de las plantas y diferentes partes de ellas, plasmando todo tipo de formas y texturas. La elasticidad y fácil colocación de los elementos vegetales en el proceso de creación, también permite direccionar cada uno de los elementos de manera flexible y rápida, así como el cambio de tonalidad. El diferente tamaño de los elementos vegetales y la elección y degradado del color, 
permite trabajar aspectos compositivos produciendo variantes expresivas.

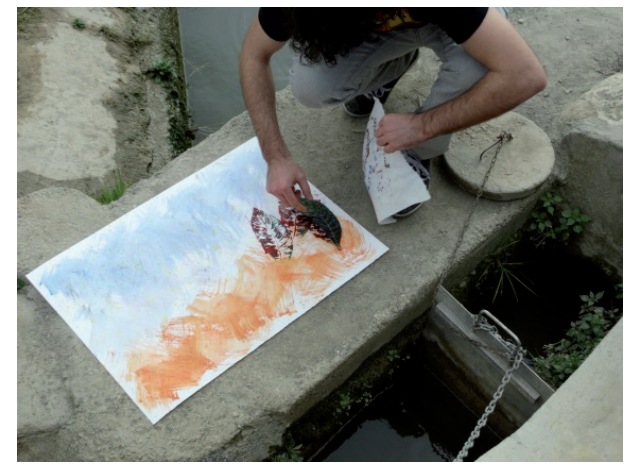

Figura 12. Proceso de realización de monotipos.

Existen otros recursos adicionales en el proceso de elaboración que también aportan riqueza plástica y expresiva, tales como: goteado (dripping), pinceladas adicionales, rasgados con espátulas, frotados con plantas, etc.

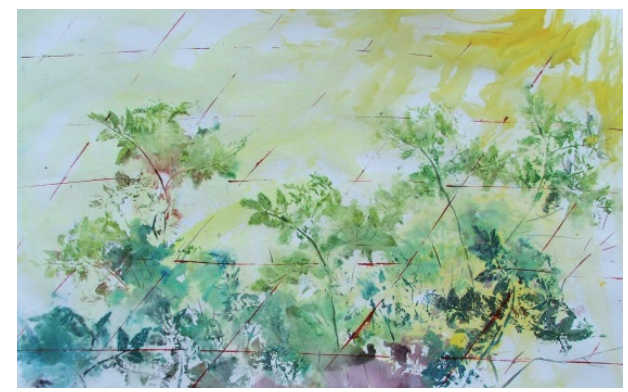

Figura 13. Estampación realizada con tallos de tomatera.

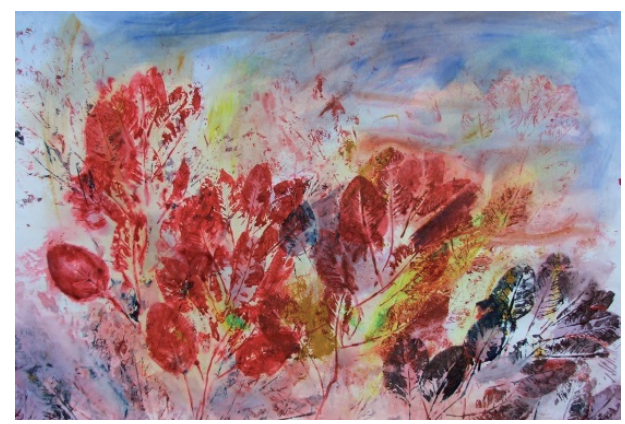

Figura 14. Estampación realizada con hojas de pimiento. 


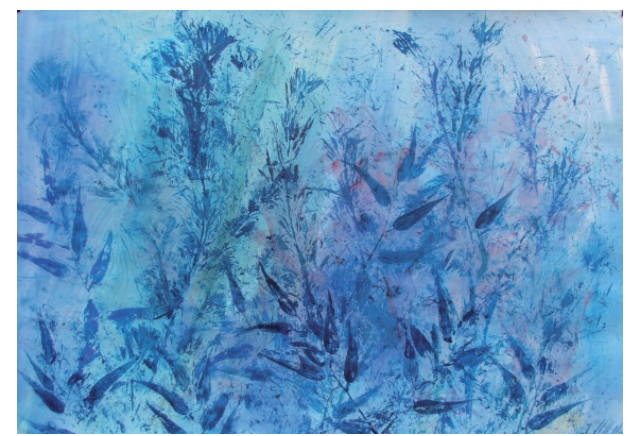

Figura 15. Estampación realizada con hojas de eucalipto.

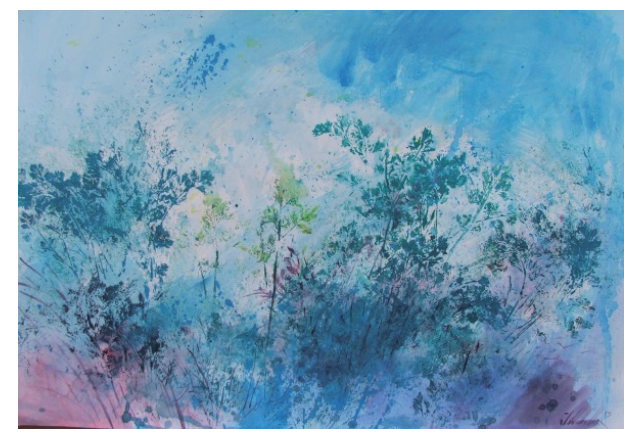

Figura 16. Estampación realizada con hojas de perejil.

\subsection{Exposición de las obras: asamblea}

El concepto expositivo lleva implícito una serie de aspectos pedagógicos que permiten crear lecturas divergentes, lo que se traduce en grandes posibilidades dialécticas que dependen de muchos factores como la experiencia del individuo, el contexto donde se sitúan las piezas protagonistas, aspectos compositivos como la proximidad de los elementos, la semejanza con otros, la continuidad de las piezas, el contraste, etc. Si analizamos cada uno de estos factores, no dejamos de pensar en las leyes de la Gestalt y su axioma principal "el todo es mayor que la suma de sus partes", que podemos utilizarlo junto a sus leyes para dar sentido a nuestra configuración mental y toda la información que nos llega a través de los canales sensoriales (Villafañe y Minguez, 2002).

Es por ello que los criterios compositivos y la relación que establecemos entre los elementos artísticos y el entorno, son fundamentales para ofrecer diferentes lecturas e incentivar el diálogo. Precisamente en 
este dialogo, el docente (guía o montador) cobra gran responsabilidad porque de su elección de elementos, distribución y manipulación se establece un recorrido vital para la percepción y posibilidades dialécticas del perceptor (alumnos en nuestro caso). La disposición e incorporación de los objetos principales con otros materiales que puedan tener connotaciones temáticas, también interfieren en este proceso cognitivo y comunicativo. Las cualidades y aspectos sensoriales de estos materiales también repercuten directamente en la percepción del individuo. Por tanto, consideramos la exposición y sus estrategias un aspecto fundamental en nuestra propuesta.

Las exposiciones se materializaron en el mismo emplazamiento donde fueron creadas las piezas, en su entorno natural, donde el alumnado ya parte de la experiencia propia del proceso creativo e interacción con sus compañeros.

El montaje de las piezas se realizó utilizando la altura que nos ofrecían los árboles y otros elementos como piedras y el propio suelo. De los árboles se colgaban finas cuerdas de esparto que comunicaban unos con otros. Las ramas descendentes también eran utilizadas como soporte para las obras. Con respecto al nivel del suelo, algunas de las producciones fueron colocadas de manera horizontal y otras de manera vertical. En las primeras, se utilizaban pequeñas piedras y maderas que estaban parcialmente superpuestas. En las segundas, se plegaban formando sencillos prismas (cuadrados, rectangulares, cilindros o trapezoidales). En conjunto, todas las piezas marcaban direcciones visuales creando un recorrido natural entre las creaciones que paradójicamente han sido creadas con los propios elementos.

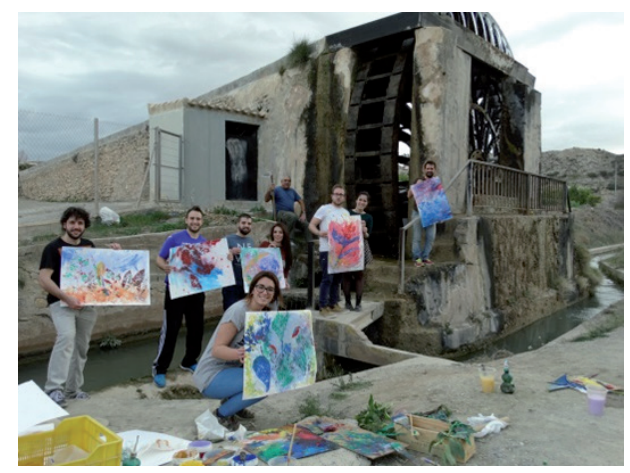

Figura 17. Exposición de las obras. 


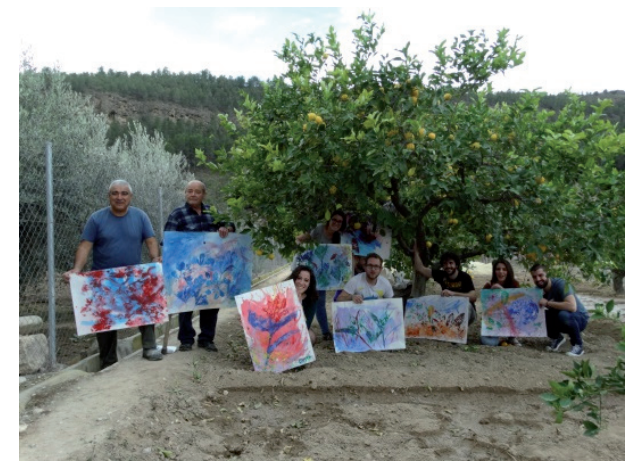

Figura 18. Exposición de obras.

\subsection{Aspectos curriculares de Educación Primaria}

Sin previa comunicación a los alumnos, se relaciona el proyecto con diversos contenidos de cinco áreas de conocimiento propias del currículum que establece los contenidos mínimos de la enseñanza en Educación Primaria. Estas áreas son: Ciencias de la Naturaleza, Matemáticas, Lengua y Literatura, Educación Física, Educación Artística, y Valores Sociales y Cívicos.

\section{Resultados y discusión}

El proyecto planteado en la metodología se ha llevado a cabo con 30 participantes divididos en dos grupos. Los resultados son los siguientes:

Los resultados se analizan en dos vertientes: Aspectos curriculares y resultados plásticos.

Con respecto a los aspectos curriculares, todos los alumnos han llevado a cabo el proyecto relacionando la actividad con más de cuatro áreas de conocimiento diferentes, incluyendo la Educación Artística. El trayecto realizado por la zona natural hasta llegar al punto de creación, provocó reacciones en el alumnado que se traducían en relaciones de la propuesta planteada con contenidos del currículum, competencias y valores. La necesidad de conocer los tipos de plantas despertó en el alumnado gran curiosidad por descubrir los tipos y procesos de cultivo, preguntando directamente a los agricultores que invitaban a 
los discentes a entrar en sus fincas. El vínculo creado entre alumnos y profesores ha generado un clima de diálogo y de pequeñas demostraciones agrícolas.

En segundo lugar, con respecto a los resultados plásticos, los alumnos trabajaron con numerosos recursos que brindaban los materiales recogidos en el trayecto, e incluso algunos no planteados inicialmente, incluyendo elementos vegetales u otros a modo de collages, realizando perforaciones y modificaciones irregulares de los formatos.

En el proceso creativo, ningún alumno mostró dificultades puesto que tenían gran curiosidad por trabajar de manera plástica con aquellas materias elementales y cotidianas, por descubrir las formas y texturas de tallos y hojas traducidas en color y estampadas en papel. Todos los alumnos manifestaron gran afecto por sus creaciones finales, incluyendo aquellos alumnos que antes de comenzar la actividad práctica mostraban cierto rechazo ante los procesos de creación plástica.

En la asamblea final, los alumnos manifestaron una serie de ventajas e inconvenientes:

Ventajas:

1- Aplicación múltiple de numerosos contenidos del currículo de Educación Primaria.

2- Flexibilidad con respecto a la posibilidad de crear diferentes adaptaciones curriculares para niños de Educación Primaria.

3- El contacto con naturaleza incentiva el proceso de aprendizaje, creando curiosidad por descubrir y conocer la realidad natural.

4- El proceso creativo y de elaboración con elementos naturales seleccionados y recogidos en el propio entorno, provoca la consolidación de experiencias favoreciendo especialmente la adquisición de contenidos y la educación en valores.

5-Adaptación del proyecto a zonas naturales muy diversas.

Inconvenientes:

1- Necesidad de personal de apoyo al profesorado para velar por los posibles peligros del entorno.

2- Necesidad de controlar las acciones del alumnado para no dañar ni alterar el entorno: instalaciones, flora y fauna.

3- Posibles reacciones alérgicas de los participantes.

4- Posibles dificultades en la asiduidad del proyecto a consecuencia de costes en materiales y en transporte. 
Didáctica artística y medioambiental: la estampación de materias vegetales como recurso educativo

José Víctor Villalba Gómez

5- Alta implicación y motivación del profesorado, que no siempre es evidente.

\section{Conclusiones}

A modo de conclusión, con esta investigación se ha comprobado que este tipo de proyectos amplía las posibilidades didácticas, permite grandes opciones para relacionar diferentes áreas de conocimiento, al mismo tiempo que permite fundir los contenidos con la realidad medioambiental y el entorno físico más próximo. Como adelantábamos en el apartado 1 (Educación Ambiental y Centros de Interpretación), esta propuesta coincide con la definición y líneas de actuación de los centros de interpretación porque hace partícipe al visitante del propio conocimiento, invitándolo a descubrir el contenido a través de su interactuación en diversas actividades. Además, la propuesta planteada motiva e integra al participante mediante acciones relacionadas con los conocimientos, impulsando un ambiente creativo en la presente propuesta mediante la manipulación controlada de especies botánicas procedentes del entorno medioambiental, que son examinadas cuidadosamente y algunas de ellas usadas como herramienta en el proceso de estampación. Las actividades propuestas implican el manejo de competencias artísticas y medioambientales contempladas en el actual currículo de Educación Primaria.

La búsqueda de nuevas propuestas estéticas a partir elementos extraídos y trabajados directamente de la naturaleza, estimula y desarrolla la creatividad de los participantes invitándoles a buscar nuevos caminos, nuevas formas expresivas, valorando y respetando el medio, además de fomentando la comunicación entre los asistentes y aportando en la mayoría de los casos, soluciones y planteamientos nuevos para abordar contenidos de la forma más adaptable a la realidad sociocultural, económica y medioambiental. Este proyecto tiene continuidad con otros trabajos llevados a cabo en entornos marítimos como los parques naturales de la albufera de Valencia y del Mar Menor, donde la flora y fauna es claramente distinta y donde son el propio paisaje y sus materiales los que frecen otro tipo de posibilidades plásticas y didácticas. 
Didáctica artística y medioambiental: la estampación de materias vegetales como

recurso educativo

José Víctor Villalba Gómez

\section{Referencias}

AEEA. (2014). Asociación española de educación ambiental. Recuperado de http://www. ae-ea.es/

AA.VV. (2014). Giuseppe Pepone: Prospectiva vegetale. Florencia. Forma Edizioni.

Bernal, M. (2013). Técnicadegrabado.es: (Difusión virtual de la gráfica impresa). Recuperado de http://www.revistalatinacs.org/067/cuadernos/14CBA.pdf

Böhm, W. (2010). Historia de la pedagogía: desde Platón hasta la actualidad. Villa María. Eduvim.

Cabezas, L., López, I., Oliver, J., Campos, R. y Barbero, M. (2016). Dibujo científico: arte y naturaleza, ilustración científica, infografía, esquemática. Madrid. Cátedra.

CENEAM (2010). Un viaje por la educación ambiental en España. Recuperado de http:// www.magrama.gob.es/es/ceneam/recursos/documentos/viaje-educambiental-espana_tcm7-13605.pdf

CENEAM (2014). Ministerio de Agricultura, Alimentación y Medio Ambiente. Recuperado de http://www.magrama.gob.es/es/ceneam/quienes-somos/

Garrido, M. (2014). Grabado: procesos y técnicas. Madrid. Akal.

Minkkinen, A. (2014). Arno Rafael Minkkinen. Recuperado de http://arno-rafael-minkkinen.com

Moreno, $M^{\mathrm{a}}$., Abad, Ma . y López, L. (2011). Talleres de arte de reciclaje con materiales hospitalarios. Arte, Individuo y Sociedad, V (23), 135-151.

Palacios, A. (2006). Educación artística y ambiental: un estudio del caso británico a través del proyecto "art and the built environment". Arte, individuo y sociedad, V (18), 57-76.

Pardo, D. (2011). Gyotaku: L'âme des poissons. Concarneau. Coop Breizh.

Tatarella, F. (2014). Natural arquitectura now. New York. Princeton architectural press.

Villafañe, J. y Mínguez, N. (2002). Principios de la teoría general de la imagen. Madrid. Pirámide.

Villalba, J. y Flores, M. (2015). Educación artística y educación transversal. Madrid. Asociación Española de Educación Ambiental. 
